\title{
Fecal metabolomics and network pharmacology reveal the correlations between constipation and depression
}

Xiaojie Liu ${ }^{1,2,3^{*}}$, Huanle Liu ${ }^{1,2,3}$, Fuxiao Wei ${ }^{1,2,3}$, Di Zhao ${ }^{1,2,3}$, Yeze Wang ${ }^{1,2,3}$, Meng $\mathrm{Lv}^{1,2,3}$, Sijun Zhao ${ }^{4}$, Xuemei Qin ${ }^{1,2,3^{*}}$

1 Modern Research Center for Traditional Chinese Medicine, Shanxi University, Taiyuan, 030006, China

${ }^{2}$ Key Laboratory of Chemical Biology and Molecular Engineering of Ministry of Education, Taiyuan, 030006, China

${ }^{3}$ Key Laboratory of Effective Substances Research and Utilization in Traditional Chinese Medicine of Shanxi Province, Taiyuan, 030006, China

${ }^{4}$ Department of Pharmacology, Shanxi Institute for Food and Drug Control, Taiyuan 030001, Shanxi, China

* Correspondence to Dr. Xiaojie Liu

E-mail: liuxiaojie@sxu.edu.cn

Tel./Fax: +863517018379 , fax: +863517018379

** Co-correspondence to Prof. Xuemei Qin

E-mail: qinxm@sxu.edu.cn

Tel./Fax: +86 3517011501, fax: +86351701150 
Table of Contents

\begin{tabular}{|c|c|}
\hline Name & Caption \\
\hline Table S1 & $\begin{array}{r}\text { The constipation-related targets collected from the GeneCards database } \\
\text { (the gene targets of constipation) }\end{array}$ \\
\hline Table S2 & $\begin{array}{r}\text { The depression-related targets collected from the GeneCards database } \\
\text { (the gene targets of depression) }\end{array}$ \\
\hline Figure S1 & $\begin{array}{r}\text { The sucrose preferences of rats in the normal control group (NC) and the } \\
\text { CUMS model group (MS) }\end{array}$ \\
\hline
\end{tabular}


Table S1 The constipation-related targets collected from the GeneCards database

\begin{tabular}{|c|c|c|c|}
\hline Symbol & Taget & Description & Score \\
\hline 1 & RET & Ret Proto-Oncogene & 30.61 \\
\hline 2 & IL6 & Interleukin 6 & 27.07 \\
\hline 3 & GDNF & Glial Cell Derived Neurotrophic Factor & 24.61 \\
\hline 4 & FLNA & Filamin A & 24.16 \\
\hline 5 & NRXN1 & Neurexin 1 & 23.32 \\
\hline 6 & KIT & KIT Proto-Oncogene, Receptor Tyrosine Kinase & 22.19 \\
\hline 7 & SNCA & Synuclein Alpha & 22.15 \\
\hline 8 & EDN3 & Endothelin 3 & 21.81 \\
\hline 9 & EDNRB & Endothelin Receptor Type B & 21.15 \\
\hline 10 & $\mathrm{TH}$ & Tyrosine Hydroxylase & 21.04 \\
\hline 11 & TP53 & Tumor Protein P53 & 21 \\
\hline 12 & KRAS & KRAS Proto-Oncogene, GTPase & 20.18 \\
\hline 13 & TCF4 & Transcription Factor 4 & 20.12 \\
\hline 14 & CCR6 & C-C Motif Chemokine Receptor 6 & 19.49 \\
\hline 15 & MECP2 & Methyl-CpG Binding Protein 2 & 19.45 \\
\hline 16 & BDNF & Brain Derived Neurotrophic Factor & 19.29 \\
\hline 17 & PPOX & Protoporphyrinogen Oxidase & 18.79 \\
\hline 18 & NRTN & Neurturin & 18.79 \\
\hline 19 & MED12 & Mediator Complex Subunit 12 & 18.52 \\
\hline 20 & ZEB2 & Zinc Finger E-Box Binding Homeobox 2 & 18.03 \\
\hline 21 & CASR & Calcium Sensing Receptor & 17.5 \\
\hline 22 & TYMP & Thymidine Phosphorylase & 17.5 \\
\hline 23 & UBE3A & Ubiquitin Protein Ligase E3A & 17.42 \\
\hline 24 & AKT1 & AKT Serine/Threonine Kinase 1 & 17.3 \\
\hline 25 & MLYCD & Malonyl-CoA Decarboxylase & 17.28 \\
\hline 26 & TLR2 & Toll Like Receptor 2 & 17.19 \\
\hline 27 & NALCN & Sodium Leak Channel, Non-Selective & 17.03 \\
\hline 28 & HRAS & HRas Proto-Oncogene, GTPase & 16.96 \\
\hline 29 & CASK & Calcium/Calmodulin Dependent Serine Protein Kinase & 16.94 \\
\hline 30 & POLG & DNA Polymerase Gamma, Catalytic Subunit & 16.93 \\
\hline 31 & $\begin{array}{c}\text { VANGL } \\
1\end{array}$ & VANGL Planar Cell Polarity Protein 1 & 16.69 \\
\hline 32 & EGFR & Epidermal Growth Factor Receptor & 16.55 \\
\hline 33 & APC & APC Regulator Of WNT Signaling Pathway & 16.44 \\
\hline 34 & SOX10 & SRY-Box Transcription Factor 10 & 16.06 \\
\hline 35 & CTNNB1 & Catenin Beta 1 & 15.91 \\
\hline 36 & CCND1 & Cyclin D1 & 15.91 \\
\hline 37 & PTH & Parathyroid Hormone & 15.91 \\
\hline 38 & CHAT & Choline O-Acetyltransferase & 15.71 \\
\hline 39 & $\begin{array}{c}\text { CDKN2 } \\
\mathrm{A}\end{array}$ & Cyclin Dependent Kinase Inhibitor 2A & 15.7 \\
\hline 40 & TCF20 & Transcription Factor 20 & 15.67 \\
\hline
\end{tabular}




\begin{tabular}{|c|c|c|}
\hline 41 & CDKL5 & Cyclin Dependent Kinase Like 5 \\
\hline 42 & MIR21 & MicroRNA 21 \\
\hline 43 & MIR483 & MicroRNA 483 \\
\hline 44 & $\begin{array}{l}\text { CNTNA } \\
\text { P2 }\end{array}$ & Contactin Associated Protein 2 \\
\hline 45 & TTR & Transthyretin \\
\hline 46 & ALB & Albumin \\
\hline 47 & BRAF & B-Raf Proto-Oncogene, Serine/Threonine Kinase \\
\hline 48 & $\mathrm{~B} 2 \mathrm{M}$ & Beta-2-Microglobulin \\
\hline 49 & SMAD4 & SMAD Family Member 4 \\
\hline 50 & PIK3CA & $\begin{array}{l}\text { Phosphatidylinositol-4,5-Bisphosphate 3-Kinase Catalytic } \\
\text { Subunit Alpha }\end{array}$ \\
\hline 51 & FOXG1 & Forkhead Box G1 \\
\hline 52 & TG & Thyroglobulin \\
\hline 53 & MBD5 & Methyl-CpG Binding Domain Protein 5 \\
\hline 54 & IQSEC2 & IQ Motif And Sec7 Domain ArfGEF 2 \\
\hline 55 & WAC & WW Domain Containing Adaptor With Coiled-Coil \\
\hline 56 & COMT & Catechol-O-Methyltransferase \\
\hline 57 & GBA & Glucosylceramidase Beta \\
\hline 58 & PEX16 & Peroxisomal Biogenesis Factor 16 \\
\hline 59 & HFE & Homeostatic Iron Regulator \\
\hline 60 & MIR17 & MicroRNA 17 \\
\hline 61 & ATRX & ATRX Chromatin Remodeler \\
\hline 62 & UCHL1 & Ubiquitin C-Terminal Hydrolase L1 \\
\hline 63 & ТPO & Thyroid Peroxidase \\
\hline 64 & LRRK2 & Leucine Rich Repeat Kinase 2 \\
\hline 65 & TWNK & Twinkle MtDNA Helicase \\
\hline 66 & SST & Somatostatin \\
\hline 67 & EP300 & E1A Binding Protein P300 \\
\hline 68 & TNF & Tumor Necrosis Factor \\
\hline 69 & SHH & Sonic Hedgehog Signaling Molecule \\
\hline 70 & MEG3 & Maternally Expressed 3 \\
\hline 71 & MIR145 & MicroRNA 145 \\
\hline 72 & ERBB2 & Erb-B2 Receptor Tyrosine Kinase 2 \\
\hline 73 & DES & Desmin \\
\hline 74 & ARID1B & AT-Rich Interaction Domain 1B \\
\hline 75 & PHOX2B & Paired Like Homeobox 2B \\
\hline 76 & CREBBP & CREB Binding Protein \\
\hline 77 & MIR126 & MicroRNA 126 \\
\hline 78 & PYY & Peptide YY \\
\hline 79 & $\begin{array}{c}\text { COL17A } \\
1\end{array}$ & Collagen Type XVII Alpha 1 Chain \\
\hline 80 & PRKN & Parkin RBR E3 Ubiquitin Protein Ligase \\
\hline 81 & VIP & Vasoactive Intestinal Peptide \\
\hline
\end{tabular}




\begin{tabular}{|c|c|c|c|}
\hline 82 & MLN & Motilin & 13.4 \\
\hline 83 & MLH1 & MutL Homolog 1 & 13.3 \\
\hline 84 & MSH2 & MutS Homolog 2 & 13.3 \\
\hline 85 & GLI2 & GLI Family Zinc Finger 2 & 13.3 \\
\hline 86 & TSHR & Thyroid Stimulating Hormone Receptor & 13.3 \\
\hline 87 & VPS35 & VPS35 Retromer Complex Component & 13.19 \\
\hline 88 & MNX1 & Motor Neuron And Pancreas Homeobox 1 & 13.07 \\
\hline 89 & SLC6A3 & Solute Carrier Family 6 Member 3 & 13.05 \\
\hline 90 & $\mathrm{CCK}$ & Cholecystokinin & 13 \\
\hline 91 & ALAD & Aminolevulinate Dehydratase & 12.97 \\
\hline 92 & CHD8 & Chromodomain Helicase DNA Binding Protein 8 & 12.97 \\
\hline 93 & ACTG2 & Actin Gamma 2, Smooth Muscle & 12.96 \\
\hline 94 & HTR4 & 5-Hydroxytryptamine Receptor 4 & 12.94 \\
\hline 95 & FGFR1 & Fibroblast Growth Factor Receptor 1 & 12.78 \\
\hline 96 & $\begin{array}{c}\text { TNFRSF } \\
1 \mathrm{~A}\end{array}$ & TNF Receptor Superfamily Member 1A & 12.78 \\
\hline 97 & CPOX & Coproporphyrinogen Oxidase & 12.78 \\
\hline 98 & HTR3A & 5-Hydroxytryptamine Receptor 3A & 12.71 \\
\hline 99 & NOD2 & Nucleotide Binding Oligomerization Domain Containing 2 & 12.69 \\
\hline 100 & MIR143 & MicroRNA 143 & 12.69 \\
\hline 101 & CLCN2 & Chloride Voltage-Gated Channel 2 & 12.55 \\
\hline 102 & BRCA1 & BRCA1 DNA Repair Associated & 12.53 \\
\hline 103 & EIF4G1 & Eukaryotic Translation Initiation Factor 4 Gamma 1 & 12.52 \\
\hline 104 & HMBS & Hydroxymethylbilane Synthase & 12.5 \\
\hline 105 & MT-CO1 & Mitochondrially Encoded Cytochrome C Oxidase I & 12.43 \\
\hline 106 & CASP8 & Caspase 8 & 12.23 \\
\hline 107 & PTCH1 & Patched 1 & 12.23 \\
\hline 108 & CTLA4 & Cytotoxic T-Lymphocyte Associated Protein 4 & 12.23 \\
\hline 109 & MIR140 & MicroRNA 140 & 12.23 \\
\hline 110 & MAPT & Microtubule Associated Protein Tau & 12.23 \\
\hline 111 & PRNP & Prion Protein & 12.23 \\
\hline 112 & COL7A1 & Collagen Type VII Alpha 1 Chain & 12.23 \\
\hline 113 & DUOX2 & Dual Oxidase 2 & 12.23 \\
\hline 114 & $\begin{array}{c}\text { SLC26A } \\
4\end{array}$ & Solute Carrier Family 26 Member 4 & 12.23 \\
\hline 115 & TSHB & Thyroid Stimulating Hormone Subunit Beta & 12.23 \\
\hline 116 & CRP & C-Reactive Protein & 12.2 \\
\hline 117 & TAC1 & Tachykinin Precursor 1 & 12.13 \\
\hline 118 & $\mathrm{H} 2 \mathrm{AC} 18$ & H2A Clustered Histone 18 & 12.11 \\
\hline 119 & MEN1 & Menin 1 & 11.95 \\
\hline 120 & ECE1 & Endothelin Converting Enzyme 1 & 11.92 \\
\hline 121 & DDC & Dopa Decarboxylase & 11.84 \\
\hline 122 & MITF & Melanocyte Inducing Transcription Factor & 11.84 \\
\hline 123 & LRIG2 & Leucine Rich Repeats And Immunoglobulin Like Domains & 11.84 \\
\hline
\end{tabular}


$\begin{array}{llll}124 & \text { USP7 } & \text { Ubiquitin Specific Peptidase } 7 & 11.8\end{array}$

125 POGZ Pogo Transposable Element Derived With ZNF Domain 11.8

126 TERT Telomerase Reverse Transcriptase 11.75

$127 \begin{array}{ccc}\text { CDKN1 } & \text { Cyclin Dependent Kinase Inhibitor 1B } & 11.75\end{array}$

128 AVP Arginine Vasopressin $\quad 11.75$

129 MIR22 MicroRNA 22 11.75

$130 \quad$ MMP1 Matrix Metallopeptidase 1 11.7

131 BRCA2 BRCA2 DNA Repair Associated 11.68

132 TGFBR2 Transforming Growth Factor Beta Receptor 2

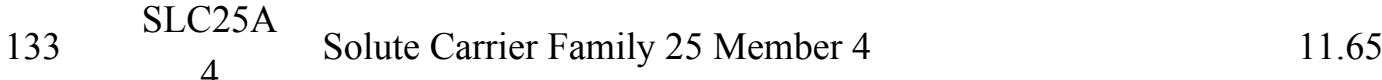

134 EPCAM Epithelial Cell Adhesion Molecule 11.65

135 SOX2 SRY-Box Transcription Factor 2 11.65

136 KCNJ1 $\begin{aligned} & \text { Potassium Inwardly Rectifying Channel Subfamily J } \\ & \text { Member 1 }\end{aligned}$

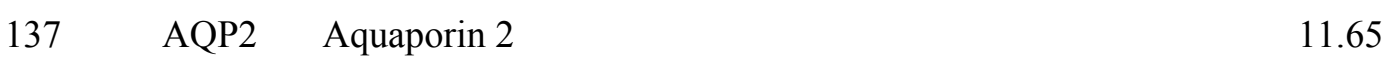

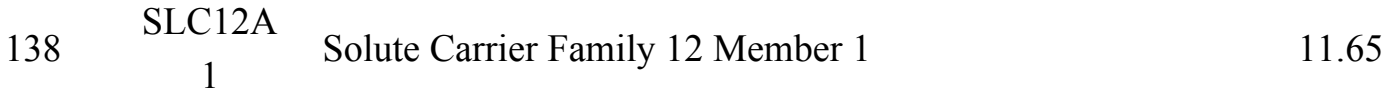

139 ASCL1 Achaete-Scute Family BHLH Transcription Factor $1 \quad 11.65$

140 POU1F1 POU Class 1 Homeobox 1 11.65

141 COQ2 Coenzyme Q2, Polyprenyltransferase $\quad 11.65$

142 SCN11A Sodium Voltage-Gated Channel Alpha Subunit $11 \quad 11.65$

143 POLG2 DNA Polymerase Gamma 2, Accessory Subunit 11.65

144 PROP1 PROP Paired-Like Homeobox 1 11.65

145 MT-TK Mitochondrially Encoded TRNA-Lys (AAA/G) 11.65

146 EBF3 EBF Transcription Factor $3 \quad 11.64$

147 GAST Gastrin 11.54

148 EGF Epidermal Growth Factor 11.45

$149 \begin{array}{ccc}\text { CDKN1 } & \text { Cyclin Dependent Kinase Inhibitor 1A } & 11.45\end{array}$

$150 \quad$ BAX $\quad$ BCL2 Associated X, Apoptosis Regulator $\quad 11.45$

$\begin{array}{llll}151 & \text { IL10 Interleukin } 10 & 11.33\end{array}$

$152 \quad$ SLC6A4 Solute Carrier Family 6 Member 4 11.27

$153 \quad$ FGF8 $\quad$ Fibroblast Growth Factor 8 11.25

$154 \begin{array}{ccc}\text { HLA-DR } & \text { Major Histocompatibility Complex, Class II, DR Beta } 1 & 11.25\end{array}$

$155 \quad$ FBN1 $\quad$ Fibrillin 1 11.25

156 NSD1 Nuclear Receptor Binding SET Domain Protein 1 11.25

157 MIR34A MicroRNA 34a 11.25

158 MIR192 MicroRNA $192 \quad 11.25$

$159 \begin{array}{ccc}\text { MIR200 } \\ \text { B }\end{array} \quad$ MicroRNA 200b 11.25 


$\begin{array}{lcll}160 & \text { MIR25 } & \text { MicroRNA 25 } & 11.25 \\ 161 & \text { MIR155 } & \text { MicroRNA 155 } & 11.25 \\ 162 & \text { MIR15A } & \text { MicroRNA 15a } & 11.25 \\ 163 & \text { CALCA } & \text { Calcitonin Related Polypeptide Alpha } & 11.24 \\ 164 & \text { SCN9A } & \text { Sodium Voltage-Gated Channel Alpha Subunit 9 } & 11.22 \\ & \text { SLC12A } & \text { Solute Carrier Family 12 Member 3 } & 11.22 \\ 165 & 3 & & 11.22 \\ 166 & \text { PAX8 } & \text { Paired Box 8 } & 11.19 \\ 167 & \text { RAI1 } & \text { Retinoic Acid Induced 1 } & 11.19 \\ 168 & \text { IL1B } & \text { Interleukin 1 Beta } & 11.03 \\ 169 & \text { MSH6 } & \text { MutS Homolog 6 } & 11.03 \\ 170 & \text { PARK7 } & \text { Parkinsonism Associated Deglycase } & 11.03 \\ 171 & \text { SLC5A5 } & \text { Solute Carrier Family 5 Member 5 } & 11.03 \\ 172 & \text { OTX2 } & \text { Orthodenticle Homeobox 2 } & 11.03 \\ 173 & \text { LMX1B } & \text { LIM Homeobox Transcription Factor 1 Beta } & 11.03 \\ 174 & \text { CDC73 } & \text { Cell Division Cycle 73 } & 11.03 \\ 175 & \text { SDHC } & \text { Succinate Dehydrogenase Complex Subunit C } & 11.03 \\ 176 & \text { NKX2-5 } & \text { NK2 Homeobox 5 } & 11.03 \\ 177 & \text { IYD } & \text { Iodotyrosine Deiodinase } & 11.03 \\ 178 & \text { SIX3 } & \text { SIX Homeobox 3 } & 11.03 \\ 179 & \text { PIGV } & \text { Phosphatidylinositol Glycan Anchor Biosynthesis Class V } & 11.03 \\ 180 & \text { TRH } & \text { Thyrotropin Releasing Hormone } & 11.03 \\ & \text { DUOXA } & \text { Dual Oxidase Maturation Factor 2 } & \end{array}$


Table S2 The depression-related targets collected from the GeneCards database

\begin{tabular}{|c|c|c|c|}
\hline Symbol & Taget & Description & Score \\
\hline 1 & SLC6A4 & Solute Carrier Family 6 Member 4 & 72.33 \\
\hline 2 & BDNF & Brain Derived Neurotrophic Factor & 66.63 \\
\hline 3 & HTR2A & 5-Hydroxytryptamine Receptor $2 \mathrm{~A}$ & 58.39 \\
\hline 4 & TPH2 & Tryptophan Hydroxylase 2 & 50.84 \\
\hline 5 & COMT & Catechol-O-Methyltransferase & 48.19 \\
\hline 6 & TNF & Tumor Necrosis Factor & 45.45 \\
\hline 7 & DRD2 & Dopamine Receptor D2 & 44.73 \\
\hline 8 & FKBP5 & FKBP Prolyl Isomerase 5 & 38.32 \\
\hline 9 & MTHFR & Methylenetetrahydrofolate Reductase & 34.68 \\
\hline 10 & CACNA1C & Calcium Voltage-Gated Channel Subunit Alpha1 C & 33.9 \\
\hline 11 & DRD3 & Dopamine Receptor D3 & 33.8 \\
\hline 12 & PCLO & Piccolo Presynaptic Cytomatrix Protein & 33.61 \\
\hline 13 & MAPT & Microtubule Associated Protein Tau & 32.47 \\
\hline 14 & HTR1A & 5-Hydroxytryptamine Receptor $1 \mathrm{~A}$ & 32.42 \\
\hline 15 & GRIN2A & Glutamate Ionotropic Receptor NMDA Type Subunit 2A & 32.39 \\
\hline 16 & $\mathrm{TH}$ & Tyrosine Hydroxylase & 32.28 \\
\hline 17 & AKT1 & AKT Serine/Threonine Kinase 1 & 32.25 \\
\hline 18 & $\mathrm{CRH}$ & Corticotropin Releasing Hormone & 32.13 \\
\hline 19 & IL10 & Interleukin 10 & 32.08 \\
\hline 20 & MAP2K1 & Mitogen-Activated Protein Kinase Kinase 1 & 31.89 \\
\hline 21 & KRAS & KRAS Proto-Oncogene, GTPase & 31.88 \\
\hline 22 & PER3 & Period Circadian Regulator 3 & 31.25 \\
\hline 23 & DISC2 & Disrupted In Schizophrenia 2 & 31.16 \\
\hline 24 & $\begin{array}{c}\text { LOC } 11080 \\
6262\end{array}$ & Solute Carrier Family 6 Member 4 Gene Promoter & 30.92 \\
\hline 25 & APOE & Apolipoprotein E & 30.76 \\
\hline 26 & IL6 & Interleukin 6 & 30.35 \\
\hline 27 & MAOA & Monoamine Oxidase A & 30.24 \\
\hline 28 & SNCA & Synuclein Alpha & 30.21 \\
\hline 29 & RELN & Reelin & 30.07 \\
\hline 30 & ESR1 & Estrogen Receptor 1 & 30 \\
\hline 31 & MECP2 & Methyl-CpG Binding Protein 2 & 29.78 \\
\hline 32 & PER2 & Period Circadian Regulator 2 & 28.89 \\
\hline 33 & MIR132 & MicroRNA 132 & 28.86 \\
\hline 34 & PRL & Prolactin & 28.54 \\
\hline 35 & GRIA1 & Glutamate Ionotropic Receptor AMPA Type Subunit 1 & 28.33 \\
\hline 36 & ALB & Albumin & 28.31 \\
\hline 37 & CRHR1 & Corticotropin Releasing Hormone Receptor 1 & 26.83 \\
\hline 38 & PRKCG & Protein Kinase C Gamma & 26.77 \\
\hline 39 & ADAM10 & ADAM Metallopeptidase Domain 10 & 26.25 \\
\hline 40 & POLG & DNA Polymerase Gamma, Catalytic Subunit & 26.18 \\
\hline 41 & FGFR1 & Fibroblast Growth Factor Receptor 1 & 26.03 \\
\hline
\end{tabular}




\begin{tabular}{|c|c|c|c|}
\hline 42 & TPH1 & Tryptophan Hydroxylase 1 & 25.57 \\
\hline 43 & $\mathrm{NR} 3 \mathrm{C} 2$ & Nuclear Receptor Subfamily 3 Group C Member 2 & 25.27 \\
\hline 44 & HTR2C & 5-Hydroxytryptamine Receptor $2 \mathrm{C}$ & 25.26 \\
\hline 45 & $\mathrm{NR} 3 \mathrm{C} 1$ & Nuclear Receptor Subfamily 3 Group C Member 1 & 25.1 \\
\hline 46 & AVP & Arginine Vasopressin & 25.01 \\
\hline 47 & SOD1 & Superoxide Dismutase 1 & 24.83 \\
\hline 48 & FMR1 & FMRP Translational Regulator 1 & 24.77 \\
\hline 49 & GNAS & GNAS Complex Locus & 24.47 \\
\hline 50 & IL1B & Interleukin 1 Beta & 24.41 \\
\hline 51 & MDD1 & Major Depressive Disorder & 24.32 \\
\hline 52 & MDD2 & Major Depressive Disorder 2 & 24.22 \\
\hline 53 & CRP & C-Reactive Protein & 24.13 \\
\hline 54 & NOS1 & Nitric Oxide Synthase 1 & 24.08 \\
\hline 55 & NRXN1 & Neurexin 1 & 23.75 \\
\hline 56 & GRIA2 & Glutamate Ionotropic Receptor AMPA Type Subunit 2 & 23.71 \\
\hline 57 & GRM1 & Glutamate Metabotropic Receptor 1 & 23.56 \\
\hline 58 & CYP2B6 & Cytochrome P450 Family 2 Subfamily B Member 6 & 23.51 \\
\hline 59 & DNMT1 & DNA Methyltransferase 1 & 23.47 \\
\hline 60 & POMC & Proopiomelanocortin & 23.06 \\
\hline 61 & PLA2G6 & Phospholipase A2 Group VI & 22.89 \\
\hline 62 & SLC6A3 & Solute Carrier Family 6 Member 3 & 22.69 \\
\hline 63 & SLC18A2 & Solute Carrier Family 18 Member A2 & 22.64 \\
\hline 64 & GRIK2 & Glutamate Ionotropic Receptor Kainate Type Subunit 2 & 22.09 \\
\hline 65 & DCTN1 & Dynactin Subunit 1 & 21.98 \\
\hline 66 & NR4A2 & Nuclear Receptor Subfamily 4 Group A Member 2 & 21.66 \\
\hline 67 & XBP1 & X-Box Binding Protein 1 & 21.62 \\
\hline 68 & CNTNAP2 & Contactin Associated Protein 2 & 21.58 \\
\hline 69 & CNR1 & Cannabinoid Receptor 1 & 21.46 \\
\hline 70 & GRIN2B & Glutamate Ionotropic Receptor NMDA Type Subunit 2B & 21.44 \\
\hline 71 & WFS1 & Wolframin ER Transmembrane Glycoprotein & 21.28 \\
\hline 72 & CREB1 & CAMP Responsive Element Binding Protein 1 & 21.23 \\
\hline 73 & SLC6A2 & Solute Carrier Family 6 Member 2 & 21.18 \\
\hline 74 & BRAF & B-Raf Proto-Oncogene, Serine/Threonine Kinase & 21.02 \\
\hline 75 & GABRA1 & $\begin{array}{l}\text { Gamma-Aminobutyric Acid Type A Receptor Subunit } \\
\text { Alpha1 }\end{array}$ & 21 \\
\hline 76 & PRNP & Prion Protein & 20.92 \\
\hline 77 & C9orf72 & C9orf72-SMCR8 Complex Subunit & 20.92 \\
\hline 78 & GRIA3 & Glutamate Ionotropic Receptor AMPA Type Subunit 3 & 20.85 \\
\hline 79 & GFAP & Glial Fibrillary Acidic Protein & 20.7 \\
\hline 80 & CACNA1A & Calcium Voltage-Gated Channel Subunit Alpha1 A & 20.64 \\
\hline 81 & PSEN1 & Presenilin 1 & 20.51 \\
\hline 82 & SHH & Sonic Hedgehog Signaling Molecule & 20.5 \\
\hline 83 & OPRM1 & Opioid Receptor Mu 1 & 20.49 \\
\hline 84 & PAH & Phenylalanine Hydroxylase & 20.47 \\
\hline
\end{tabular}




\begin{tabular}{|c|c|c|c|}
\hline 85 & GRID2 & Glutamate Ionotropic Receptor Delta Type Subunit 2 & 20.44 \\
\hline 86 & LEP & Leptin & 20.31 \\
\hline 87 & HTR3A & 5-Hydroxytryptamine Receptor 3A & 20.29 \\
\hline 88 & DAOA & D-Amino Acid Oxidase Activator & 20.26 \\
\hline 89 & FGFR2 & Fibroblast Growth Factor Receptor 2 & 20.25 \\
\hline 90 & $\mathrm{DAO}$ & D-Amino Acid Oxidase & 20.25 \\
\hline 91 & FGF8 & Fibroblast Growth Factor 8 & 20.24 \\
\hline 92 & $\mathrm{ACE}$ & Angiotensin I Converting Enzyme & 20.14 \\
\hline 93 & TWNK & Twinkle MtDNA Helicase & 20.03 \\
\hline 94 & GNRH1 & Gonadotropin Releasing Hormone 1 & 19.95 \\
\hline 95 & NTRK2 & Neurotrophic Receptor Tyrosine Kinase 2 & 19.94 \\
\hline 96 & COL11A2 & Collagen Type XI Alpha 2 Chain & 19.75 \\
\hline 97 & MAP2K2 & Mitogen-Activated Protein Kinase Kinase 2 & 19.68 \\
\hline 98 & IGF1 & Insulin Like Growth Factor 1 & 19.66 \\
\hline 99 & NPY & Neuropeptide Y & 19.57 \\
\hline 100 & DRD4 & Dopamine Receptor D4 & 19.53 \\
\hline 101 & PRKN & Parkin RBR E3 Ubiquitin Protein Ligase & 19.48 \\
\hline 102 & PRKAR1A & $\begin{array}{l}\text { Protein Kinase CAMP-Dependent Type I Regulatory } \\
\text { Subunit Alpha }\end{array}$ & 19.35 \\
\hline 103 & COL2A1 & Collagen Type II Alpha 1 Chain & 19.34 \\
\hline 104 & DLG3 & Discs Large MAGUK Scaffold Protein 3 & 19.14 \\
\hline 105 & OXT & Oxytocin/Neurophysin I Prepropeptide & 19.13 \\
\hline 106 & FGFR3 & Fibroblast Growth Factor Receptor 3 & 19.11 \\
\hline 107 & NGF & Nerve Growth Factor & 19.02 \\
\hline 108 & HRAS & HRas Proto-Oncogene, GTPase & 18.9 \\
\hline 109 & HTR1B & 5-Hydroxytryptamine Receptor 1B & 18.88 \\
\hline 110 & GDNF & Glial Cell Derived Neurotrophic Factor & 18.81 \\
\hline 111 & GRIN1 & Glutamate Ionotropic Receptor NMDA Type Subunit 1 & 18.8 \\
\hline 112 & DRD1 & Dopamine Receptor D1 & 18.79 \\
\hline 113 & GLI2 & GLI Family Zinc Finger 2 & 18.7 \\
\hline 114 & CYP2D6 & Cytochrome P450 Family 2 Subfamily D Member 6 & 18.69 \\
\hline 115 & SHANK3 & SH3 And Multiple Ankyrin Repeat Domains 3 & 18.64 \\
\hline 116 & TP53 & Tumor Protein P53 & 18.59 \\
\hline 117 & COL1A1 & Collagen Type I Alpha 1 Chain & 18.58 \\
\hline 118 & GCH1 & GTP Cyclohydrolase 1 & 18.56 \\
\hline 119 & MAPK1 & Mitogen-Activated Protein Kinase 1 & 18.36 \\
\hline 120 & HTT & Huntingtin & 18.33 \\
\hline 121 & OXTR & Oxytocin Receptor & 18.19 \\
\hline 122 & DISC1 & DISC1 Scaffold Protein & 18.04 \\
\hline 123 & GBA & Glucosylceramidase Beta & 18.01 \\
\hline 124 & GRN & Granulin Precursor & 17.98 \\
\hline 125 & $\mathrm{VCP}$ & Valosin Containing Protein & 17.93 \\
\hline 126 & SST & Somatostatin & 17.87 \\
\hline 127 & NOTCH3 & Notch Receptor 3 & 17.87 \\
\hline
\end{tabular}




\begin{tabular}{|c|c|c|c|}
\hline 128 & ATP2A2 & $\begin{array}{l}\text { ATPase Sarcoplasmic/Endoplasmic Reticulum Ca2+ } \\
\text { Transporting } 2\end{array}$ & 17.85 \\
\hline 129 & GRM5 & Glutamate Metabotropic Receptor 5 & 17.84 \\
\hline 130 & GNB3 & G Protein Subunit Beta 3 & 17.83 \\
\hline 131 & TOR1A & Torsin Family 1 Member A & 17.78 \\
\hline 132 & TARDBP & TAR DNA Binding Protein & 17.69 \\
\hline 133 & MAOB & Monoamine Oxidase B & 17.64 \\
\hline 134 & PARK7 & Parkinsonism Associated Deglycase & 17.61 \\
\hline 135 & $\begin{array}{c}\text { TMEM106 } \\
\text { B }\end{array}$ & Transmembrane Protein 106B & 17.55 \\
\hline 136 & ATP1A3 & ATPase $\mathrm{Na}+/ \mathrm{K}+$ Transporting Subunit Alpha 3 & 17.52 \\
\hline 137 & IFNG & Interferon Gamma & 17.51 \\
\hline 138 & PTEN & Phosphatase And Tensin Homolog & 17.49 \\
\hline 139 & PICK1 & Protein Interacting With PRKCA 1 & 17.49 \\
\hline 140 & GSK3B & Glycogen Synthase Kinase 3 Beta & 17.46 \\
\hline 141 & HCRT & Hypocretin Neuropeptide Precursor & 17.34 \\
\hline 142 & GABRD & $\begin{array}{l}\text { Gamma-Aminobutyric Acid Type A Receptor Subunit } \\
\text { Delta }\end{array}$ & 17.28 \\
\hline 143 & GRM2 & Glutamate Metabotropic Receptor 2 & 17.27 \\
\hline 144 & LRRK2 & Leucine Rich Repeat Kinase 2 & 17.25 \\
\hline 145 & WASHC5 & WASH Complex Subunit 5 & 17.23 \\
\hline 146 & GLI3 & GLI Family Zinc Finger 3 & 17.16 \\
\hline 147 & COL11A1 & Collagen Type XI Alpha 1 Chain & 17.14 \\
\hline 148 & ARSA & Arylsulfatase A & 17.11 \\
\hline 149 & CHMP2B & Charged Multivesicular Body Protein 2B & 17.1 \\
\hline 150 & PTCH1 & Patched 1 & 17.09 \\
\hline 151 & CHAT & Choline O-Acetyltransferase & 17.06 \\
\hline 152 & POU1F1 & POU Class 1 Homeobox 1 & 17.05 \\
\hline 153 & ALG9 & ALG9 Alpha-1,2-Mannosyltransferase & 17.02 \\
\hline 154 & NR1D1 & Nuclear Receptor Subfamily 1 Group D Member 1 & 17.01 \\
\hline 155 & ATP13A2 & ATPase Cation Transporting 13A2 & 16.96 \\
\hline 156 & CLOCK & Clock Circadian Regulator & 16.94 \\
\hline 157 & FLNA & Filamin A & 16.92 \\
\hline 158 & SYN2 & Synapsin II & 16.85 \\
\hline 159 & NPAS2 & Neuronal PAS Domain Protein 2 & 16.77 \\
\hline 160 & SYP & Synaptophysin & 16.76 \\
\hline 161 & ACTB & Actin Beta & 16.73 \\
\hline 162 & CBLN1 & Cerebellin 1 Precursor & 16.72 \\
\hline 163 & INS & Insulin & 16.71 \\
\hline 164 & $\begin{array}{l}\text { HLA-DRB } \\
\quad 1\end{array}$ & Major Histocompatibility Complex, Class II, DR Beta 1 & 16.69 \\
\hline 165 & GAD1 & Glutamate Decarboxylase 1 & 16.64 \\
\hline 166 & SGCE & Sarcoglycan Epsilon & 16.55 \\
\hline 167 & MTOR & Mechanistic Target Of Rapamycin Kinase & 16.52 \\
\hline
\end{tabular}


Twist Family BHLH Transcription Factor 2

Parathyroid Hormone

Solute Carrier Family 1 Member 2

Purinergic Receptor P2X 7

Discs Large MAGUK Scaffold Protein 1

Zic Family Member 2

Lysine Methyltransferase 2D

Dystrobrevin Binding Protein 1

HTR1D

5-Hydroxytryptamine Receptor 1D 


\begin{tabular}{|c|c|c|c|}
\hline 211 & VEGFA & Vascular Endothelial Growth Factor A & 15.12 \\
\hline 212 & GRM3 & Glutamate Metabotropic Receptor 3 & 15.12 \\
\hline 213 & ARNTL & Aryl Hydrocarbon Receptor Nuclear Translocator Like & 15.11 \\
\hline 214 & PAX6 & Paired Box 6 & 15.06 \\
\hline 215 & ATRX & ATRX Chromatin Remodeler & 15.06 \\
\hline 216 & GAL & Galanin And GMAP Prepropeptide & 15.03 \\
\hline 217 & TGIF1 & TGFB Induced Factor Homeobox 1 & 15.03 \\
\hline 218 & $\mathrm{CP}$ & Ceruloplasmin & 15.01 \\
\hline 219 & TBX1 & T-Box Transcription Factor 1 & 15 \\
\hline 220 & DISP1 & Dispatched RND Transporter Family Member 1 & 14.98 \\
\hline 221 & NF1 & Neurofibromin 1 & 14.97 \\
\hline 222 & HUWE1 & $\begin{array}{l}\text { HECT, UBA And WWE Domain Containing E3 Ubiquitin } \\
\text { Protein Ligase } 1\end{array}$ & 14. \\
\hline 223 & PDLIM5 & PDZ And LIM Domain 5 & 14.93 \\
\hline 224 & CYP2C19 & Cytochrome P450 Family 2 Subfamily C Member 19 & 14.93 \\
\hline 225 & RRM2B & $\begin{array}{l}\text { Ribonucleotide Reductase Regulatory TP53 Inducible } \\
\text { Subunit M2B }\end{array}$ & 14. \\
\hline 226 & MAFD2 & Major Affective Disorder 2 & 14.86 \\
\hline 227 & ATXN8OS & ATXN8 Opposite Strand LncRNA & 14.83 \\
\hline 228 & OTX2 & Orthodenticle Homeobox 2 & 14.78 \\
\hline 229 & ADCYAP1 & Adenylate Cyclase Activating Polypeptide 1 & 14.75 \\
\hline 230 & FBN1 & Fibrillin 1 & 14.73 \\
\hline 231 & ZNF804A & Zinc Finger Protein 804A & 14.7 \\
\hline 232 & SLC25A4 & Solute Carrier Family 25 Member 4 & 14.59 \\
\hline 233 & CRY1 & Cryptochrome Circadian Regulator 1 & 14.54 \\
\hline 234 & CRY2 & Cryptochrome Circadian Regulator 2 & 14.53 \\
\hline 235 & ANK3 & Ankyrin 3 & 14.49 \\
\hline 236 & ALX4 & ALX Homeobox 4 & 14.45 \\
\hline 237 & $\mathrm{XK}$ & X-Linked Kx Blood Group & 14.41 \\
\hline 238 & INPPL1 & Inositol Polyphosphate Phosphatase Like 1 & 14.35 \\
\hline 239 & SIX3 & SIX Homeobox 3 & 14.32 \\
\hline 240 & ELN & Elastin & 14.28 \\
\hline 241 & SETD5 & SET Domain Containing 5 & 14.25 \\
\hline 242 & CYP1A2 & Cytochrome P450 Family 1 Subfamily A Member 2 & 14.2 \\
\hline 243 & NRAS & NRAS Proto-Oncogene, GTPase & 14.2 \\
\hline 244 & CLN6 & CLN6 Transmembrane ER Protein & 14.18 \\
\hline 245 & JPH3 & Junctophilin 3 & 14.11 \\
\hline 246 & PRODH & Proline Dehydrogenase 1 & 14.05 \\
\hline 247 & TAC1 & Tachykinin Precursor 1 & 13.96 \\
\hline 248 & CYP3A4 & Cytochrome P450 Family 3 Subfamily A Member 4 & 13.96 \\
\hline 249 & SLC1A3 & Solute Carrier Family 1 Member 3 & 13.88 \\
\hline 250 & CASR & Calcium Sensing Receptor & 13.87 \\
\hline 251 & TSPO & Translocator Protein & 13.86 \\
\hline 252 & UCHL1 & Ubiquitin C-Terminal Hydrolase L1 & 13.8 \\
\hline
\end{tabular}




\begin{tabular}{|c|c|c|c|}
\hline 253 & POLG2 & DNA Polymerase Gamma 2, Accessory Subunit & 13.77 \\
\hline 254 & CTLA4 & Cytotoxic T-Lymphocyte Associated Protein 4 & 13.7 \\
\hline 255 & MAFD3 & Major Affective Disorder 3, Early Onset & 13.67 \\
\hline 256 & MAFD4 & Major Affective Disorder 4 & 13.67 \\
\hline 257 & MAFD5 & Major Affective Disorder 5 & 13.67 \\
\hline 258 & MAFD6 & Major Affective Disorder 6 & 13.67 \\
\hline 259 & MAFD8 & Major Affective Disorder-8, Susceptibility To & 13.67 \\
\hline 260 & MAFD9 & Major Affective Disorder-9, Susceptibility To & 13.67 \\
\hline 261 & KMT2A & Lysine Methyltransferase 2A & 13.62 \\
\hline 262 & DMD & Dystrophin & 13.58 \\
\hline 263 & GABRG2 & $\begin{array}{l}\text { Gamma-Aminobutyric Acid Type A Receptor Subunit } \\
\text { Gamma2 }\end{array}$ & 13.58 \\
\hline 264 & $\mathrm{CDON}$ & Cell Adhesion Associated, Oncogene Regulated & 13.54 \\
\hline 265 & ARID1B & AT-Rich Interaction Domain 1B & 13.53 \\
\hline 266 & ATXN10 & Ataxin 10 & 13.53 \\
\hline 267 & CRLF1 & Cytokine Receptor Like Factor 1 & 13.52 \\
\hline 268 & SMPD1 & Sphingomyelin Phosphodiesterase 1 & 13.49 \\
\hline 269 & CYP2C9 & Cytochrome P450 Family 2 Subfamily C Member 9 & 13.45 \\
\hline 270 & TREM2 & Triggering Receptor Expressed On Myeloid Cells 2 & 13.42 \\
\hline 271 & LBR & Lamin B Receptor & 13.39 \\
\hline 272 & SMARCA4 & $\begin{array}{l}\text { SWI/SNF Related, Matrix Associated, Actin Dependent } \\
\text { Regulator Of Chromatin, Subfamily A, Member } 4\end{array}$ & 13.39 \\
\hline 273 & DVL3 & Dishevelled Segment Polarity Protein 3 & 13.37 \\
\hline 274 & NSD1 & Nuclear Receptor Binding SET Domain Protein 1 & 13.35 \\
\hline 275 & $\mathrm{DBH}$ & Dopamine Beta-Hydroxylase & 13.32 \\
\hline 276 & ZBTB20 & Zinc Finger And BTB Domain Containing 20 & 13.24 \\
\hline 277 & HLA-A & Major Histocompatibility Complex, Class I, A & 13.23 \\
\hline 278 & IL2 & Interleukin 2 & 13.22 \\
\hline 279 & ADRA2A & Adrenoceptor Alpha 2A & 13.18 \\
\hline 280 & BMP2 & Bone Morphogenetic Protein 2 & 13.17 \\
\hline 281 & FOXG1 & Forkhead Box G1 & 13.14 \\
\hline 282 & PIK3R1 & Phosphoinositide-3-Kinase Regulatory Subunit 1 & 13.14 \\
\hline 283 & H2AC18 & H2A Clustered Histone 18 & 13.11 \\
\hline 284 & BMPER & BMP Binding Endothelial Regulator & 13.07 \\
\hline 285 & TACR3 & Tachykinin Receptor 3 & 13.06 \\
\hline 286 & MAN2B1 & Mannosidase Alpha Class 2B Member 1 & 13.06 \\
\hline 287 & RUNX2 & RUNX Family Transcription Factor 2 & 13.05 \\
\hline 288 & PTH1R & Parathyroid Hormone 1 Receptor & 13.05 \\
\hline 289 & EP300 & E1A Binding Protein P300 & 13.01 \\
\hline 290 & SIRT1 & Sirtuin 1 & 12.96 \\
\hline 291 & S100B & S100 Calcium Binding Protein B & 12.95 \\
\hline 292 & DLL1 & Delta Like Canonical Notch Ligand 1 & 12.92 \\
\hline 293 & FOXH1 & Forkhead Box H1 & 12.92 \\
\hline 294 & VPS13A & Vacuolar Protein Sorting 13 Homolog A & 12.9 \\
\hline
\end{tabular}




\begin{tabular}{|c|c|c|c|}
\hline 295 & C19orf12 & Chromosome 19 Open Reading Frame 12 & 12.9 \\
\hline 296 & HSPG2 & Heparan Sulfate Proteoglycan 2 & 12.83 \\
\hline 297 & MED13L & Mediator Complex Subunit 13L & 12.8 \\
\hline 298 & FAM20C & FAM20C Golgi Associated Secretory Pathway Kinase & 12.77 \\
\hline 299 & STXBP1 & Syntaxin Binding Protein 1 & 12.75 \\
\hline 300 & LRP8 & LDL Receptor Related Protein 8 & 12.75 \\
\hline 301 & PPP2R2B & Protein Phosphatase 2 Regulatory Subunit Bbeta & 12.75 \\
\hline 302 & ADAMTS2 & $\begin{array}{l}\text { ADAM Metallopeptidase With Thrombospondin Type } 1 \\
\text { Motif } 2\end{array}$ & 12.74 \\
\hline 303 & ZBTB24 & Zinc Finger And BTB Domain Containing 24 & 12.74 \\
\hline 304 & $\mathrm{SCN} 2 \mathrm{~A}$ & Sodium Voltage-Gated Channel Alpha Subunit 2 & 12.7 \\
\hline 305 & FADS1 & Fatty Acid Desaturase 1 & 12.69 \\
\hline 306 & RYR1 & Ryanodine Receptor 1 & 12.68 \\
\hline 307 & TP63 & Tumor Protein P63 & 12.65 \\
\hline 308 & TFAP2B & Transcription Factor AP-2 Beta & 12.61 \\
\hline 309 & GPC6 & Glypican 6 & 12.59 \\
\hline 310 & MED12 & Mediator Complex Subunit 12 & 12.58 \\
\hline 311 & PSEN2 & Presenilin 2 & 12.57 \\
\hline 312 & LIPA & Lipase A, Lysosomal Acid Type & 12.55 \\
\hline 313 & CDKL5 & Cyclin Dependent Kinase Like 5 & 12.55 \\
\hline 314 & HSD17B4 & Hydroxysteroid 17-Beta Dehydrogenase 4 & 12.52 \\
\hline 315 & SLC26A2 & Solute Carrier Family 26 Member 2 & 12.5 \\
\hline 316 & FA2H & Fatty Acid 2-Hydroxylase & 12.5 \\
\hline 317 & CLIP1 & CAP-Gly Domain Containing Linker Protein 1 & 12.5 \\
\hline 318 & COQ2 & Coenzyme Q2, Polyprenyltransferase & 12.5 \\
\hline 319 & PDE4D & Phosphodiesterase 4D & 12.47 \\
\hline 320 & HFE & Homeostatic Iron Regulator & 12.45 \\
\hline 321 & SQSTM1 & Sequestosome 1 & 12.44 \\
\hline 322 & CRHBP & Corticotropin Releasing Hormone Binding Protein & 12.43 \\
\hline 323 & JAG1 & Jagged Canonical Notch Ligand 1 & 12.42 \\
\hline 324 & MT-CO1 & Mitochondrially Encoded Cytochrome C Oxidase I & 12.41 \\
\hline 325 & KISS1 & KiSS-1 Metastasis Suppressor & 12.41 \\
\hline 326 & PDE11A & Phosphodiesterase 11A & 12.41 \\
\hline 327 & GNA11 & G Protein Subunit Alpha 11 & 12.39 \\
\hline 328 & EDAR & Ectodysplasin A Receptor & 12.38 \\
\hline 329 & COASY & Coenzyme A Synthase & 12.34 \\
\hline 330 & CRHR2 & Corticotropin Releasing Hormone Receptor 2 & 12.33 \\
\hline 331 & RAB39B & RAB39B, Member RAS Oncogene Family & 12.3 \\
\hline 332 & ACTG1 & Actin Gamma 1 & 12.29 \\
\hline 333 & GPX4 & Glutathione Peroxidase 4 & 12.27 \\
\hline 334 & NTF3 & Neurotrophin 3 & 12.26 \\
\hline 335 & $\mathrm{MEF} 2 \mathrm{C}$ & Myocyte Enhancer Factor 2C & 12.26 \\
\hline 336 & KISS1R & KISS1 Receptor & 12.26 \\
\hline 337 & PDYN & Prodynorphin & 12.23 \\
\hline
\end{tabular}




\begin{tabular}{|c|c|c|c|}
\hline 338 & CD96 & CD96 Molecule & 12.2 \\
\hline 339 & GABBR1 & Gamma-Aminobutyric Acid Type B Receptor Subunit 1 & 12.19 \\
\hline 340 & GAS1 & Growth Arrest Specific 1 & 12.14 \\
\hline 341 & PTPN22 & Protein Tyrosine Phosphatase Non-Receptor Type 22 & 12.09 \\
\hline 342 & TMEM216 & Transmembrane Protein 216 & 12.08 \\
\hline 343 & TBX4 & T-Box Transcription Factor 4 & 12.08 \\
\hline 344 & GABRB3 & $\begin{array}{l}\text { Gamma-Aminobutyric Acid Type A Receptor Subunit } \\
\text { Beta3 }\end{array}$ & 12.06 \\
\hline 345 & HESX1 & HESX Homeobox 1 & 12.06 \\
\hline 346 & HDAC4 & Histone Deacetylase 4 & 12.04 \\
\hline 347 & HTR2B & 5-Hydroxytryptamine Receptor 2B & 12.03 \\
\hline 348 & FUS & FUS RNA Binding Protein & 12.01 \\
\hline 349 & STAG2 & Stromal Antigen 2 & 11.89 \\
\hline 350 & SOS1 & SOS Ras/Rac Guanine Nucleotide Exchange Factor 1 & 11.89 \\
\hline 351 & CISD2 & CDGSH Iron Sulfur Domain 2 & 11.89 \\
\hline 352 & MIR140 & MicroRNA 140 & 11.88 \\
\hline 353 & PIGY & Phosphatidylinositol Glycan Anchor Biosynthesis Class Y & 11.87 \\
\hline 354 & MTNR1A & Melatonin Receptor 1A & 11.86 \\
\hline 355 & GLA & Galactosidase Alpha & 11.84 \\
\hline 356 & GNRHR & Gonadotropin Releasing Hormone Receptor & 11.83 \\
\hline 357 & RET & Ret Proto-Oncogene & 11.83 \\
\hline 358 & ARID2 & AT-Rich Interaction Domain 2 & 11.8 \\
\hline 359 & GLUL & Glutamate-Ammonia Ligase & 11.8 \\
\hline 360 & COL1A2 & Collagen Type I Alpha 2 Chain & 11.8 \\
\hline 361 & WAC & WW Domain Containing Adaptor With Coiled-Coil & 11.79 \\
\hline 362 & CXCL8 & C-X-C Motif Chemokine Ligand 8 & 11.78 \\
\hline 363 & PDGFRB & Platelet Derived Growth Factor Receptor Beta & 11.76 \\
\hline 364 & NRG1 & Neuregulin 1 & 11.76 \\
\hline 365 & PRKCA & Protein Kinase C Alpha & 11.74 \\
\hline 366 & FKBP4 & FKBP Prolyl Isomerase 4 & 11.72 \\
\hline 367 & NKX2-5 & NK2 Homeobox 5 & 11.72 \\
\hline 368 & ITPR1 & Inositol 1,4,5-Trisphosphate Receptor Type 1 & 11.72 \\
\hline 369 & DVL1 & Dishevelled Segment Polarity Protein 1 & 11.71 \\
\hline 370 & MT-TK & Mitochondrially Encoded TRNA-Lys (AAA/G) & 11.69 \\
\hline 371 & GPR50 & G Protein-Coupled Receptor 50 & 11.68 \\
\hline 372 & TEF & TEF Transcription Factor, PAR BZIP Family Member & 11.67 \\
\hline 373 & DMPK & DM1 Protein Kinase & 11.66 \\
\hline 374 & $\mathrm{NPC} 1$ & NPC Intracellular Cholesterol Transporter 1 & 11.65 \\
\hline 375 & B3GAT3 & Beta-1,3-Glucuronyltransferase 3 & 11.65 \\
\hline 376 & DGUOK & Deoxyguanosine Kinase & 11.64 \\
\hline 377 & ADNP & Activity Dependent Neuroprotector Homeobox & 11.63 \\
\hline 378 & DRD5 & Dopamine Receptor D5 & 11.62 \\
\hline 379 & IMPA1 & Inositol Monophosphatase 1 & 11.59 \\
\hline 380 & MT-ND1 & Mitochondrially Encoded NADH:Ubiquinone & 11.5 \\
\hline
\end{tabular}


Oxidoreductase Core Subunit 1

Potassium Inwardly Rectifying Channel Subfamily J

Member 2

Paired Like Homeodomain 2

Mitochondrially Encoded NADH:Ubiquinone

Oxidoreductase Core Subunit 4

LHPP

Phospholysine Phosphohistidine Inorganic Pyrophosphate Phosphatase

RNA Component Of Mitochondrial RNA Processing

EDA

Ectodysplasin A

PIK3CA

Phosphatidylinositol-4,5-Bisphosphate 3-Kinase Catalytic Subunit Alpha

CHI3L1

Chitinase 3 Like 1

Transient Receptor Potential Cation Channel Subfamily V

\section{DYNC2H1 Dynein Cytoplasmic 2 Heavy Chain 1}

Erb-B2 Receptor Tyrosine Kinase 4

SWI/SNF Related, Matrix Associated, Actin Dependent 


$\begin{array}{lclc}416 & \text { XYLT1 } & \text { Xylosyltransferase 1 } & 11.24 \\ 417 & \text { SLC17A5 } & \text { Solute Carrier Family 17 Member 5 } & 11.22 \\ 418 & \text { EIF2B5 } & \text { Eukaryotic Translation Initiation Factor 2B Subunit Epsilon } & 11.21 \\ 419 & \text { SIN3A } & \text { SIN3 Transcription Regulator Family Member A } & 11.2 \\ 420 & \text { KAT6B } & \text { Lysine Acetyltransferase 6B } & 11.19 \\ 421 & \text { PIGL } & \text { Phosphatidylinositol Glycan Anchor Biosynthesis Class L } & 11.18 \\ 422 & \text { HMBS } & \text { Hydroxymethylbilane Synthase } & 11.17 \\ 423 & \text { PLOD1 } & \text { Procollagen-Lysine,2-Oxoglutarate 5-Dioxygenase 1 } & 11.16 \\ 424 & \text { SCN9A } & \text { Sodium Voltage-Gated Channel Alpha Subunit 9 } & 11.16 \\ 425 & \text { PLCB1 } & \text { Phospholipase C Beta 1 } & 11.15 \\ 426 & \text { HDAC8 } & \text { Histone Deacetylase 8 } & 11.14 \\ 427 & \text { IQSEC2 } & \text { IQ Motif And Sec7 Domain ArfGEF 2 } & 11.14 \\ 428 & \text { EHMT1 } & \text { Euchromatic Histone Lysine Methyltransferase 1 } & 11.13 \\ 429 & \text { PROKR2 } & \text { Prokineticin Receptor 2 } & 11.12 \\ 430 & \text { SNAP25 } & \text { Synaptosome Associated Protein 25 } & 11.11 \\ 431 & \text { IFNA1 } & \text { Interferon Alpha 1 } & 11.11 \\ 432 & \text { TONSL } & \text { Tonsoku Like, DNA Repair Protein } & 11.11 \\ 433 & \text { NEK1 } & \text { NIMA Related Kinase 1 } & 11.08 \\ 434 & \text { PROP1 } & \text { PROP Paired-Like Homeobox 1 } & 11.08 \\ 435 & \text { SMC3 } & \text { Structural Maintenance Of Chromosomes 3 } & 11.08 \\ 436 & \text { STIN2-VN } & \text { Serotonin Transporter Intronic VNTR Enhancer } & 11.07 \\ 437 & \text { TR } & \text { TACR2 } & \text { Tachykinin Receptor 2 } \\ 438 & \text { CPOX } & \text { Coproporphyrinogen Oxidase } & 11.07 \\ 439 & \text { CLCF1 } & \text { Cardiotrophin Like Cytokine Factor 1 } & 11.04 \\ 440 & \text { PIEZO2 } & \text { Piezo Type Mechanosensitive Ion Channel Component 2 } & 11.03 \\ & & & 1.02\end{array}$




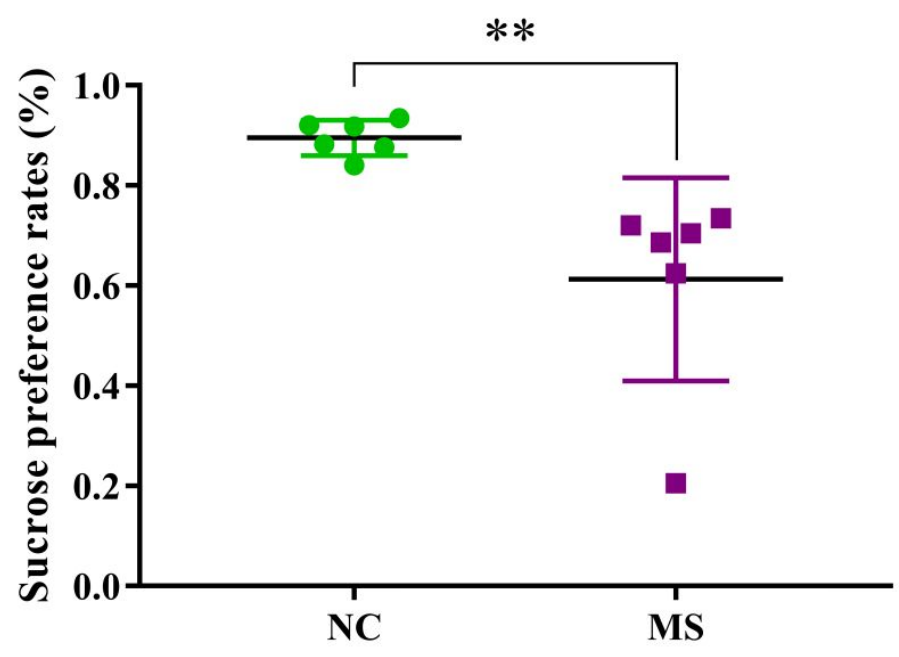

Figure S1 The sucrose preferences of rats in the normal control group (NC) and the CUMS model group (MS). 